\title{
A Grand Challenge. 2. Phenotypic Profiling of a Natural Product Library on Parkinson's Patient-Derived Cells
}

\author{
Marie-Laure Vial, ${ }^{\dagger}$ Dusan Zencak, ${ }^{\dagger}$ Tanja Grkovic, ${ }^{\dagger}$ Alain-Dominique Gorse, ${ }^{\ddagger}$ Alan Mackay-Sim, ${ }^{\dagger}$ \\ George D. Mellick, ${ }^{\dagger}$ Stephen A. Wood, ${ }^{* \dagger}$ and Ronald J. Quinn ${ }^{*}{ }^{\dagger}$ \\ 'Eskitis Institute for Drug Discovery, Griffith University, Brisbane, QLD 4111, Australia \\ ${ }^{\ddagger}$ QFAB Bioinformatics, Institute for Molecular Bioscience, The University of Queensland, St Lucia, QLD 4072, Australia
}

\section{Supporting Information}

\begin{abstract}
Harnessing the inherent biological relevance of natural products requires a method for the recognition of biological effects that may subsequently lead to the discovery of particular targets. An unbiased multidimensional profiling method was used to examine the activities of natural products on primary cells derived from a Parkinson's disease patient. The biological signature of 482 natural products was examined using multiparametric analysis to investigate known cellular pathways and organelles implicated in Parkinson's disease such as mitochondria, lysosomes, endosomes, apoptosis, and autophagy. By targeting several cell components simultaneously the chance of finding a phenotype was increased. The phenotypes were then clustered using an uncentered correlation. The

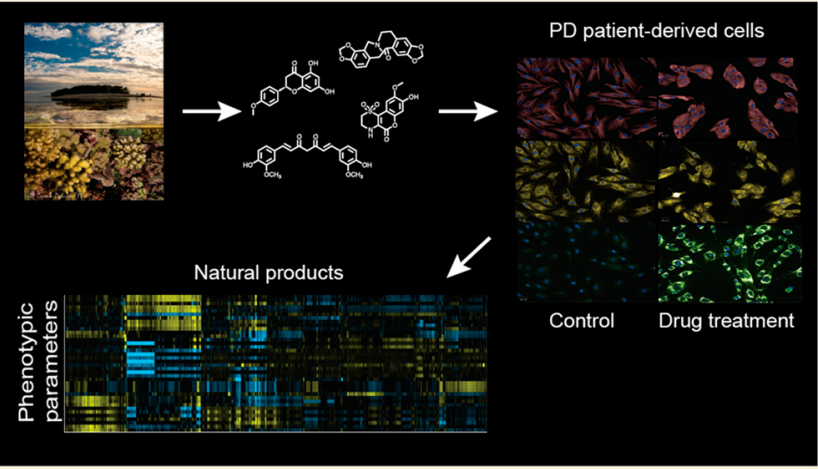
multidimensional phenotypic screening showed that all natural products, in our screening set, were biologically relevant compounds as determined by an observed phenotypic effect. Multidimensional phenotypic screening can predict the cellular function and subcellular site of activity of new compounds, while the cluster analysis provides correlation with compounds with known mechanisms of action. This study reinforces the value of natural products as biologically relevant compounds.
\end{abstract}

\begin{abstract}
A s part of a research program aiming to address a grand challenge in natural product chemistry to determine the function of all natural products, a set of 482 natural products was analyzed using a phenotypic screen. Natural products are validated starting points for drug design, and compound libraries based on natural product starting points have largely succeeded. ${ }^{1-3}$ It has been proposed that the large compound libraries used in high-throughput screening (HTS) may not reflect the rich diversity of a smaller, purified compound natural products library. ${ }^{4}$

Harnessing the inherent biological relevance of natural products ${ }^{5}$ requires a method for the recognition of biological function that may subsequently lead by correlation to the discovery of particular targets. HTS assays developed for drug discovery purposes can be either target- or cell-based. ${ }^{6}$ Focused screening against a single target of interest requires a large screening set and is frequently associated with a low validated hit rate. Cell-based approaches facilitate investigation of an entire pathway and can be used to investigate networks. Cellbased assays are not a novel concept in drug discovery; however they usually target a specific pathway or are restricted to one or only a few organelles. In the past decade, several studies have demonstrated the potential of cytological profiling to interrogate the biological responses elicited by compound libraries. ${ }^{7}$ These cell-based screening methods have almost exclusively been performed on immortalized cells such as HeLa cells ${ }^{8,9}$ or
\end{abstract}

U2OS cells. ${ }^{10}$ In addition, the fluorescent probes used targeted specific proteins such as SC35, phospho-p38, anillin, c-Fos, ${ }^{9}$ or only a few organelles. ${ }^{11}$ The study reported here differs from these previous phenotypic profiling methods by investigating six independent cellular compartments on a patient-derived cell model in order to identify phenotypes. By targeting multiple cellular compartments, such as mitochondria, lysosomes, cytoskeleton, endosomes, autophagosomes, and nuclei, the chances of finding a phenotypic response are increased. We performed the method on a nontransformed/nonimmortalized primary cell line as it might represent normal physiological responses on a patient-derived model. Nontransformed and nonimmortalized primary human olfactory neurospherederived (hONS) cells with a normal karyotype, derived from a patient with idiopathic Parkinson's disease, model the disease. $^{12-14}$ This cell model displays a large cytoplasmic/ nuclear ratio, enhancing the sensitivity to alterations in the shape, size, and localization of cytoplasmic organelles.

In previous research, iotrochotazine A (1) and jaspamycin (2) exhibited different effects on lysosome and early endosome parameters compared with vehicle, suggesting that these compound have an activity on vesicular trafficking in patientderived hONS cells from a patient with idiopathic Parkinson's

Received: March 22, 2016

Published: July 22, 2016 
disease. ${ }^{14,15}$ Herein, 482 natural products were screened on the patient-derived cell model to identify compounds that induce a unique profile, to cluster the phenotypes by their cytological signature, and to determine if chemical similarity drives a biological response.<smiles>COc1cc2c3c(c(=O)oc2cc1O)NCCS3(=O)=O</smiles><smiles>N#Cc1cn([C@@H]2OC(CO)[C@@H](O)[C@H]2O)c2nc[nH]c(=O)c12</smiles>

\section{RESULTS AND DISCUSSION}

Fluorescent probes were used to simultaneously stain nuclei and the microtubule-based cytoskeleton of hONS cells to detect changes in overall cell morphology and cytoarchitecture in addition to cellular pathways and organelles implicated in Parkinson's disease such as mitochondria, lysosomes, endosomes, and autophagy on a patient-derived cell model of Parkinson's disease. ${ }^{12,13}$ Compounds were distributed robotically to two separate 384-well plates, with each plate subjected to a different set of dyes. Stain set 1 contained a DNA stain (4',6-diamidino-2-phenylindole, Dapi) ${ }^{16}$ an anti-early endosome antigen 1 (EEAl) antibody to mark early endosomes, ${ }^{17}$ LysoTracker Red DND-99, a fluorescent dye highly selective for acidic organelles, ${ }^{18}$ particularly lysosomes, and CellMask to stain the plasma membrane ${ }^{19}$ and hence report cell area. Stain set 2 included Dapi, an anti- $\alpha$-tubulin antibody to mark the microtubules $^{20}$ and reflect cell area, MitoTracker Orange CMTMRos, a fluorescent dye, to stain mitochondria, ${ }^{21}$ and an anti-microtubule-associated protein 1 light chain $3 b$ (LC3b) antibody to label autophagosomes. ${ }^{22}$ These two stain sets examined six cellular components with Dapi used in both sets to define the nuclei and to examine nuclear morphology. Images were collected automatically on a high-content imaging system using a $20 \times$ high numerical aperture objective lens to capture high-resolution images from multiple cells per image. Six images per well, for each fluorescent channel, were collected, resulting in at least 100 cells analyzed for each compound. Individual cells were segmented based on the Dapi nuclear stain, using the "Find Nuclei" building block, and the microtubules stain or CellMask, using the "Find Cytoplasm" building block, in the Harmony High Content Imaging and Analysis Software in order to quantify cellular parameters that change after compound exposure (Figure 1).

From the combination of different fluorescent probes, 38 parameters, encompassing cellular, nuclear, and organelle morphology, staining intensity, and texture throughout the cell and in different subcellular regions, were generated (Supporting Information, Table S1). Data were collected and averaged from segmented images of individual cells and wells (at least 100 cells were scored per treatment). To assess the sensitivity and reproducibility of the assay, both of the imaging and hONS cell response, a three-probe set of molecules, including nocodazole, ${ }^{23}$ chloroquine, ${ }^{24}$ and rotenone, ${ }^{25}$ which disrupt microtubules, lysosomes, and mitochondrial function, respectively, was used in at least 10 technical replicates and four biological replicates. These data confirmed this approach was
Stain set 1

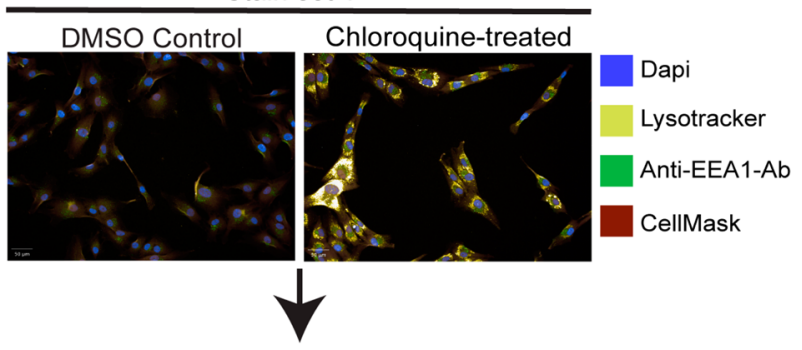

Image segmentation, detection and quantification of changes after drug exposure

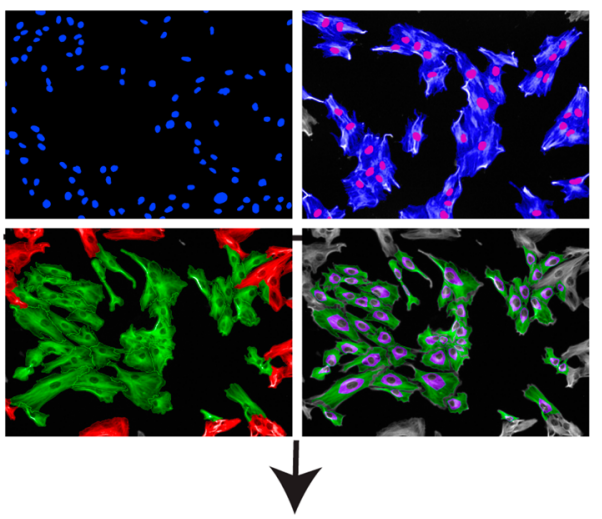

38 cytological parameters

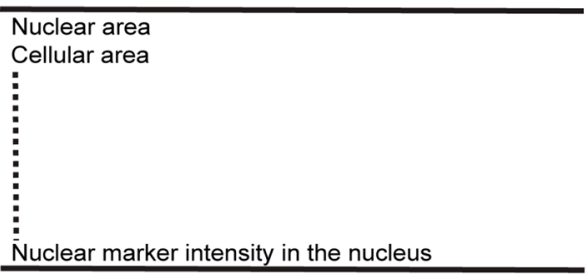

Figure 1. Large-scale multidimensional profiling strategy using stain set 1 . Compounds were distributed using an automated system in an optically clear bottom CellCarrier 384-well plate. Parkinson's disease hONS cells were added to compounds for final concentrations of 1,3 , 10 , and $30 \mu \mathrm{M}(0.6 \% \mathrm{DMSO})$ and incubated for $24 \mathrm{~h}$. Stain set 1 contained a DNA marker (Dapi in blue), a cytoskeletal marker (CellMask in deep red), a lysosomal marker (LysoTracker Red DND99 in yellow), and an endosomal marker (anti-EEA1 antibody in green). Automated fluorescence microscopy was carried out using the high-content imaging system Operetta (PerkinElmer), and images were acquired using a $20 \times$ objective. Six images per well for each wavelength were collected. Representative images are shown with 50 $\mu \mathrm{m}$ size bars. Stain set 2 was used in a similar procedure to generate, after individual cell segmentation, 38 parameters for each compound treatment.

viable and robust for the identification of compounds with biological activities (Figure 2).

The multidimensional screening platform was used to profile the biological activity of 590 pure natural products including 45 new or novel compounds. The compounds were derived from Nature Bank, ${ }^{26}$ a natural product library, which includes biologically active small molecules, as well as new and novel compounds. Tanimoto similarity scores ${ }^{27}$ showed that the compound library contains chemically diverse compounds. At Tanimoto constants (Tc) of 0.6, 0.5, and 0.4, the 590 natural products were clustered into 419,352 , and 274 chemical similarity clusters, respectively. Each of the 590 natural products was profiled in four concentrations $(1,3,10$, and 30 $\mu \mathrm{M})$ to assess a dose-response. On every plate, 24 wells 
a

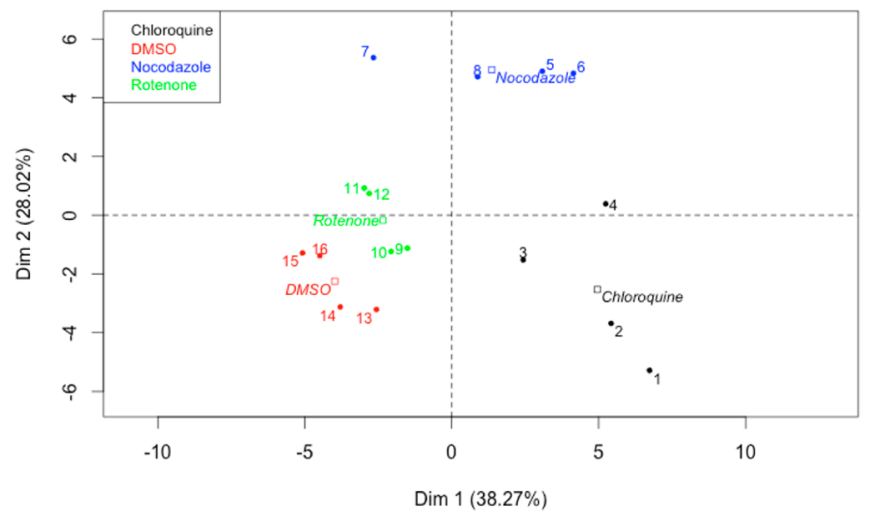

b

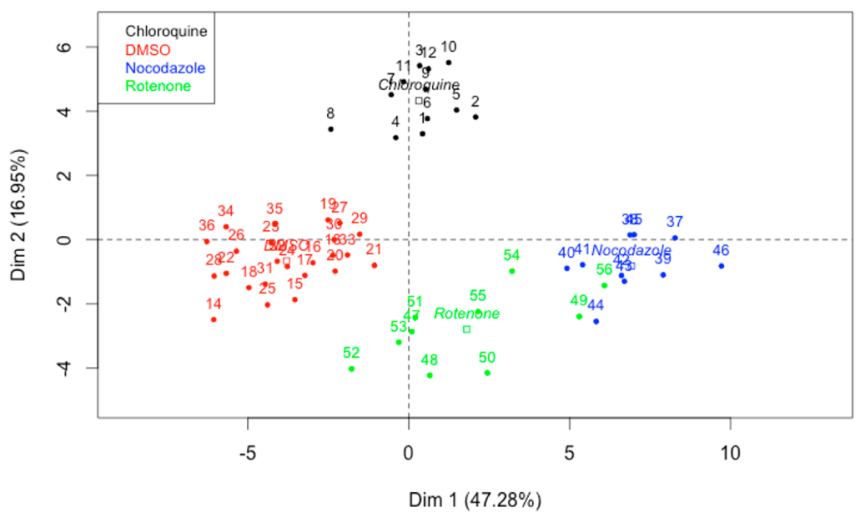

Figure 2. Principal component analysis (PCA) plot for sensitivity and reproducibility assessment of the assay. (a) PCA of four biological replicates after treatment with rotenone ( $20 \mu \mathrm{M}, 0.6 \% \mathrm{DMSO})$, chloroquine (10 $\mu \mathrm{M}, 0.6 \% \mathrm{DMSO})$, nocodazole $(10 \mu \mathrm{M}, 0.6 \% \mathrm{DMSO})$, and the control ( $0.6 \%$ DMSO). The first two principal components explained $66 \%$ of the variance. (b) PCA of at least 10 technical replicates after treatment with rotenone (20 $\mu \mathrm{M}, 0.6 \% \mathrm{DMSO})$, chloroquine (10 $\mu \mathrm{M}, 0.6 \% \mathrm{DMSO})$, nocodazole (10 $\mu \mathrm{M}, 0.6 \% \mathrm{DMSO})$, and the control (0.6\% DMSO). The first two principal components explained $64 \%$ of the variance.

contained dimethyl sulfoxide (DMSO) only, to provide data on the normal biological variation in the cultured hONS cells exposed to vehicle. The variation of control wells did not exceed $13 \%$ for all parameters investigated. The low variability between the 24 replicates confirmed the robustness of the screening method. These data established the baseline for evaluation of compound activity.

Following screening, highly cytotoxic natural products, which prevented scoring of at least 100 cells, were excluded from the data. The phenotypic changes were scored in several steps. First, the normality of the distribution of all parameters was checked and $\log _{2}$ transformation was calculated in order to obtain a normal distribution for outliers (Supporting Information Figure S1a, b, and c). Significant phenotypic perturbations following compound exposure when compared with the negative control were identified by a $t$ test. The biological signature of natural products was determined by calculating a $z$-score for each parameter, which compares each compound to the mean of all natural products profiled. The $z$ score was set at 0 when not significantly different from DMSO $(p>0.05)$. The factor scores were clustered based on the pairwise uncentered correlation coefficients using Cluster 3.0 and visualized as a dendrogram via Java Treeview. The dendrogram contained 482 compounds and gave 13 prominent bioclusters, containing at least 20 natural products, based on the similarity of their biological profile (uncentered correlation $>0.7$ ) (Figure 3a). The 13 bioclusters contained 459 natural products, including 35 new or novel compounds (Supporting Information Table S2). Colchicine, ${ }^{28}$ cytochalasin $\mathrm{D},{ }^{29}$ and paclitaxel, ${ }^{30}$ compounds that have an effect on the cytoskeleton, were representative of biocluster 8 (Figure $3 c$ and $d$ ). The data for each biocluster are shown in Supporting Information Table S2. Biological signatures of protopine, also reported as an antibacterial, $^{31}$ ajmaline, an antiarrhythmic agent, ${ }^{32}$ and gitoxigenin, an antineoplastic agent, ${ }^{33}$ at different concentrations, are shown in Figure $3 \mathrm{~b}$. Protopine was found in biocluster 7 at 1,3 , and $10 \mu \mathrm{M}$, whereas protopine at $30 \mu \mathrm{M}$ was in cluster 8. Ajmaline had all its four concentrations in cluster 8 , and gitoxigenin was found in biocluster 7 at $1 \mu \mathrm{M}$, whereas this compound was in cluster 8 from a concentration of $3 \mu \mathrm{M}$ (Figure $3 \mathrm{~b}$ ). Our results indicate that phenotypic profile is linked to concentration.

We observed that $39 \%$ of the compounds had all four concentrations in the same phenotypic cluster. Additionally, $89 \%$ of the natural products had at least three concentrations in the same biocluster. For some natural products, a phenotype was observed only at or above $3 \mu \mathrm{M}$; consequently, the $1 \mu \mathrm{M}$ concentration was clustered apart from the others. In some cases, compounds exhibited a strong phenotype at $30 \mu \mathrm{M}$; therefore this concentration did not cluster with 1,3 , and 10 $\mu \mathrm{M}$. Our results show the importance of performing doseresponse assays to characterize in detail the biological activity of compounds and also to determine the appropriate concentration for further analysis.

Compounds in biocluster 1 and in biocluster 6 predominantly exhibited a decrease in the intensity of the lysosomal marker, LysoTracker. In contrast, an increase in the LysoTracker intensity was predominant in bioclusters 7, 8, 9, 10, and 11 (Figure 4a). Carminic acid at $10 \mu \mathrm{M}$, which was associated with biocluster 1 , induced a negative deviation from the mean in the intensity of the lysosomal marker, LysoTracker. E6 berbamine at $1 \mu \mathrm{M}$ was included in biocluster 7 and exhibited a positive deviation from the mean in the intensity of the LysoTracker (Figure 4b). Bioclusters 2, 8, 11, and 12 contained natural products exhibiting a marked effect on cellular morphology. Natural products in biocluster 2 induced a positive deviation from the mean in cell area, while natural products associated with bioclusters 8,11 , and 12 induced a decrease in cell area (Figure $4 \mathrm{c}$ ). Tomatidine at $10 \mu \mathrm{M}$ was contained in biocluster 8 and exhibited a negative deviation from the mean in cell area. Conversely, etoposide at $3 \mu \mathrm{M}$, which was associated with biocluster 2, showed a positive deviation from the mean in cell area (Figure $4 \mathrm{~d}$ ). Compounds in bioclusters 3 and 4 shared a similar profile to biocluster 2 but with a lesser effect and without changes in cell morphology. Early endosomal features were a predominant alteration in most of the bioclusters. Bioclusters 10 and 13 were associated with natural products mainly inducing a negative deviation from the mean in the number and intensity of EEA1-labeled early endosomes. Conversely, small molecules included in bioclusters $2,7,8$, and 9 resulted in a positive deviation in the 
a

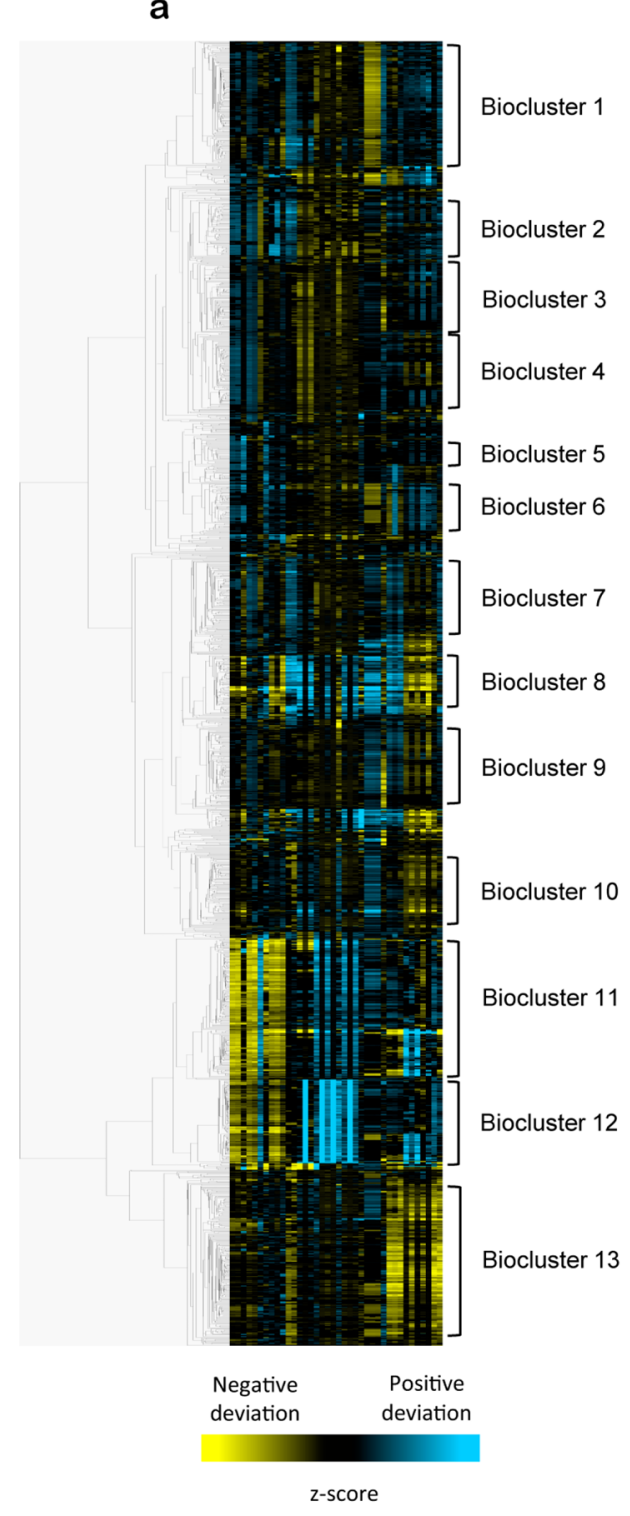

C

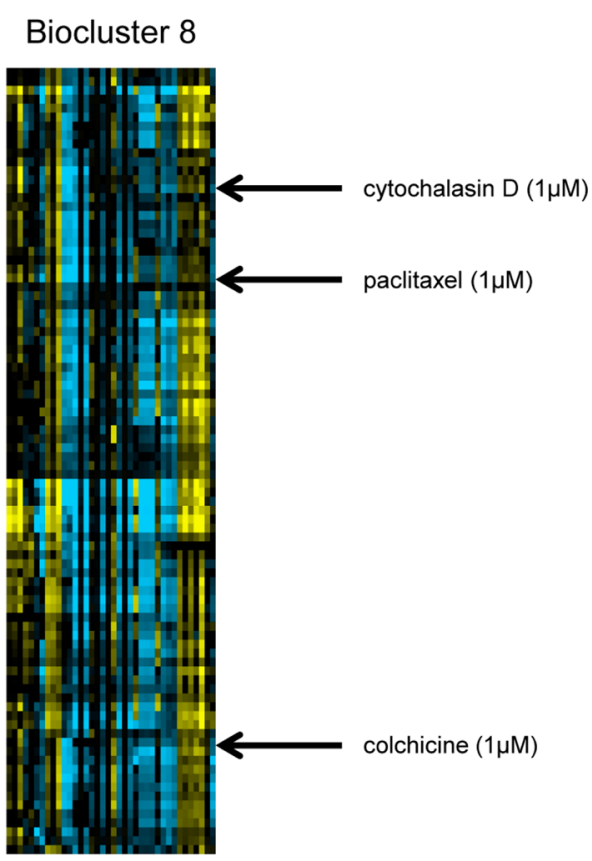

d
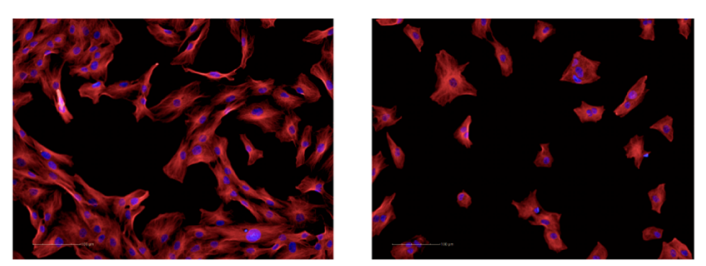

$0.6 \%$ DMSO

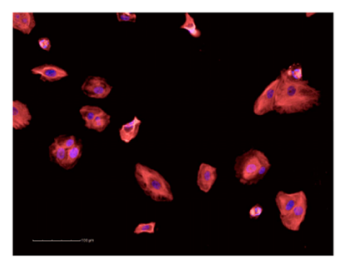

paclitaxel $(1 \mu \mathrm{M})$

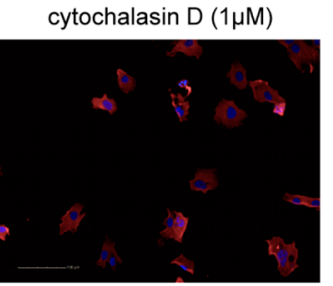

colchicine $(1 \mu \mathrm{M})$

b

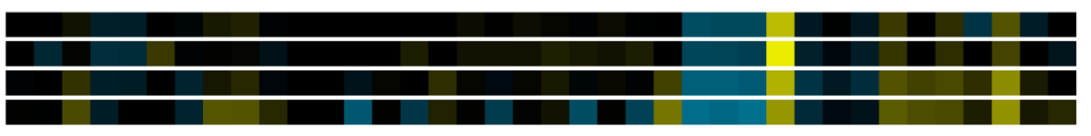

protopine $1 \mu \mathrm{M}$ (biocluster 7 ) protopine $3 \mu \mathrm{M}$ (biocluster 7 ) protopine $10 \mu \mathrm{M}$ (biocluster 7 ) protopine $30 \mu \mathrm{M}$ (biocluster 8 )

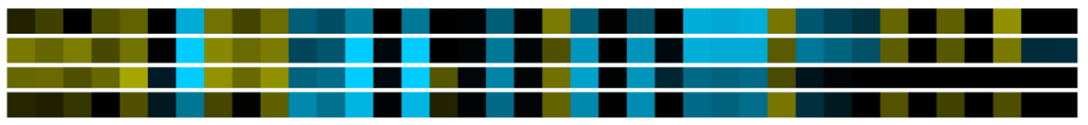

ajmaline $1 \mu \mathrm{M}$ (biocluster 8 ) ajmaline $3 \mu \mathrm{M}$ (biocluster 8 ) ajmaline $10 \mu \mathrm{M}$ (biocluster 8 ) ajmaline $30 \mu \mathrm{M}$ (biocluster 8 )

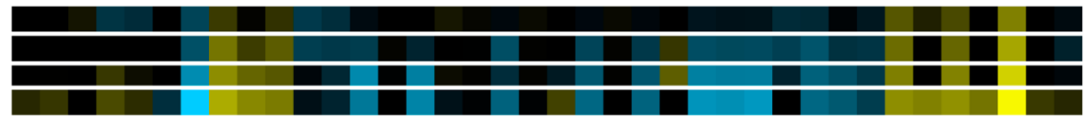

gitoxigenin $1 \mu \mathrm{M}$ (biocluster 7 ) gitoxigenin $3 \mu \mathrm{M}$ (biocluster 8 )

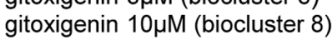

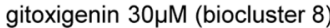

Figure 3. Heatmap depicting the cytological profile of natural products, based on $z$-score calculations comparing each compound to the mean of all natural products, with pertinent clusters highlighted. (a) Complete data set of 482 unique samples from Nature Bank. Compounds were hierarchically clustered based on their pairwise uncentered correlation coefficients, and 13 clusters with an uncentered correlation coefficient superior to 0.7 were defined from the dendrogram. Uncentered correlation coefficient/cluster: $0.767 / 1 ; 0.739 / 2 ; 0.730 / 3 ; 0.728 / 4 ; 0.761 / 5 ; 0.764 / 6 ; 0.715 /$ $7 ; 0.775 / 8 ; 0.743 / 9 ; 0.731 / 10 ; 0.764 / 11 ; 0.729 / 12$; and $0.756 / 13$. Individual compounds are presented on the $y$-axis with individual features on the $x$-axis. (b) View of biocluster 8 with the phenotypic signature of cytochalasin D, paclitaxel, and colchicine, at $1 \mu \mathrm{M}(0.6 \% \mathrm{DMSO})$, highlighted. (c) Representative images of Parkinson's disease hONS cells treated with $0.6 \%$ DMSO, cytochalasin D, paclitaxel, and colchicine at $1 \mu \mathrm{M}(0.6 \%$ DMSO) for $24 \mathrm{~h}$ and stained for nuclei and $\alpha$-tubulin (deep red). (d) Dose-response activities. Biological profiles of protopine, ajmaline, and gitoxigenin at 1, 3,10 , and $30 \mu \mathrm{M}(0.6 \%$ DMSO) on 38 parameters. 


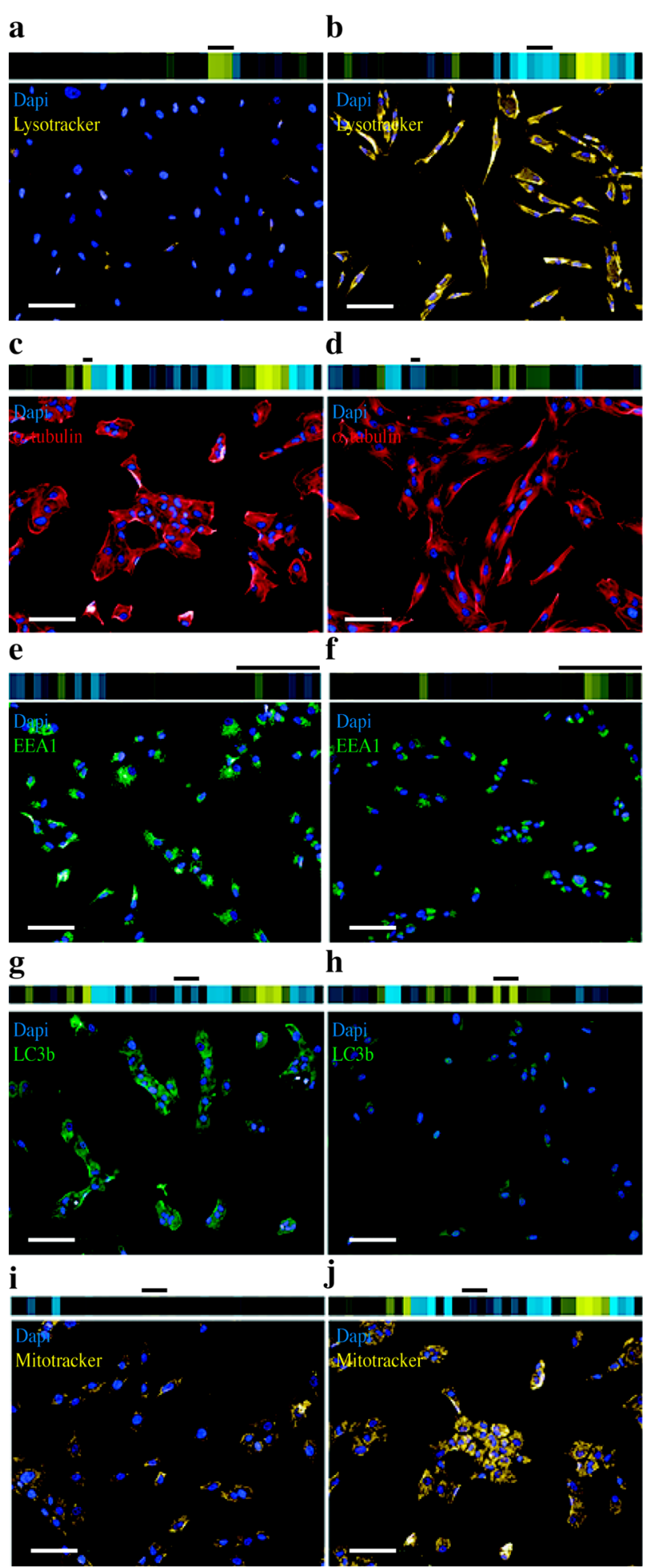

Figure 4. Representative profiles and images of compounds affecting different cellular markers. (a) Carminic acid at $10 \mu \mathrm{M}$ exhibited a negative deviation from the mean of all compounds in the intensity of LysoTracker (Biocluster 1). (b) E6 berbamine at $1 \mu \mathrm{M}$ exhibited a positive deviation from the mean of all compounds in intensity of LysoTracker (biocluster 7). (c) Tomatidine at $10 \mu \mathrm{M}$ showed a negative deviation from the mean of all compounds in cell area (biocluster 8). (d) Etoposide at $3 \mu \mathrm{M}$ exhibited a positive deviation from the mean of all compounds in cell area (biocluster 2). (e) Baicalein at $30 \mu \mathrm{M}$ showed a decrease in number of EEA1 marker associated with a positive deviation from the mean of all compounds in intensity and texture of EEA1 marker (biocluster 2). (f) Cafestol
Figure 4. continued

acetate at $10 \mu \mathrm{M}$ exhibited a negative deviation from the mean of all compounds for all EEA1 features (biocluster 10). (g) Antibiotic A23187 at $1 \mu \mathrm{M}$ showing an increase in intensity of LC3b marker (biocluster 8). (h) Forskolin at $10 \mu \mathrm{M}$ exhibited a negative deviation from the mean of all compounds in LC3b marker intensity (biocluster 2). (i) Bromocriptine mesylate at $1 \mu \mathrm{M}$ showed a slight negative deviation from the mean of all compounds in MitoTracker intensity (biocluster 5). (j) Tomatidine at $10 \mu \mathrm{M}$ exhibited a positive deviation from the mean of all compounds in MitoTracker intensity (biocluster 8). Black bars above biological signatures represent LysoTracker intensity ( $a$ and b), cell area ( $c$ and d), EEAl marker features (e and f), LC3b marker intensity ( $g$ and $h$ ), and MitoTracker intensity ( $i$ and $j$ ). Cells were seeded at 1350 cells/well in growth medium, treated for 24 $\mathrm{h}$, and imaged with a $20 \times$ high numerical aperture objective on the Operetta (PerkinElmer). Scale bars $=100 \mu \mathrm{m}$.

intensity of EEA1 marker. Figure $4 \mathrm{e}$ and $\mathrm{f}$ present an example of compounds showing an alteration of EEA1-labeled early endosomes. Baicalein at $30 \mu \mathrm{M}$, which was associated with biocluster 2, induced a decrease in the number of EEA1 marker associated with a positive deviation from the mean in intensity and texture of the EEA1 marker (Figure 4e). Cafestol acetate, at $10 \mu \mathrm{M}$, was included in biocluster 10 and showed a negative deviation from the mean for all EEA1 features (Figure 4f). Bioclusters 8, 11, and 12 were constituted of compounds, such as antibiotic A-23187 at $1 \mu \mathrm{M}$ (biocluster 8 ), showing a positive deviation in the intensity of the LC3b marker (Figure $4 \mathrm{~g}$ ). In contrast, natural products associated within biocluster 2 including forskolin at $10 \mu \mathrm{M}$ exhibited a slight decrease in the intensity of the LC3b marker (Figure $4 h$ ). Figure $4 i$ and $j$ present an example of compounds inducing a modification in the mitochondrial marker, MitoTracker. Bromocriptine mesylate at $1 \mu \mathrm{M}$ was associated with biocluster 5 and exhibited a slight negative deviation from the mean in MitoTracker intensity (Figure 4i). Conversely, tomatidine at $10 \mu \mathrm{M}$ was associated with biocluster 8 and exhibited a positive deviation from the mean in MitoTracker intensity (Figure 4j). Biocluster 5 was characterized by a decrease in Dapi intensity. In contrast, bioclusters 11 and 12 contained compounds showing an increase in Dapi intensity. However, natural products in biocluster 11 induced a lesser effect than those in biocluster 12 .

The chemical diversity within the 13 bioclusters was analyzed for all compounds that had all four concentrations in the same biocluster to avoid bias due to a dose-response activity. The data for biocluster 1 were typical of all bioclusters. Compounds in this phenotypic biocluster showed mainly a negative deviation from the mean of all natural products tested for lysosomal features. The similarity search was performed on the 22 compounds that had all four concentrations in this phenotypic cluster. At a Tc of 0.6 , only three compounds were grouped together (Figure 5a), and a Tc of 0.5 and 0.4 revealed three chemical similarity groups (Figure $5 b$ ). At a Tc of $0.3,15$ compounds were included in five chemical similarity groups (Figure 5c) and seven natural products remained singletons (Figure $5 \mathrm{~d}$ ). The results of the structure similarity assessed using a Tc of 0.3 for bioclusters 1 to 13 are shown in the Supporting Information Table S3. For eight bioclusters, no structure similarity was observed with a Tc between 0.9 and 0.6 . Chemical similarity clearly does not drive the biological response. Rather, the chemical similarity analysis showed that several chemical classes were represented in each biocluster to produce a similar phenotype. Compounds with different 
$\mathbf{a}$<smiles></smiles>

b
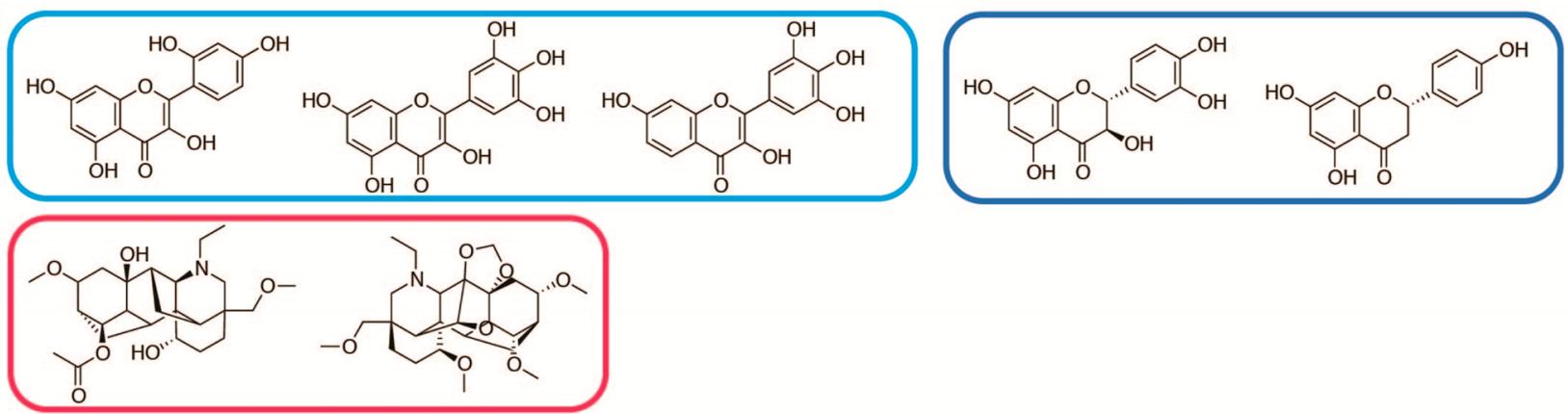

c
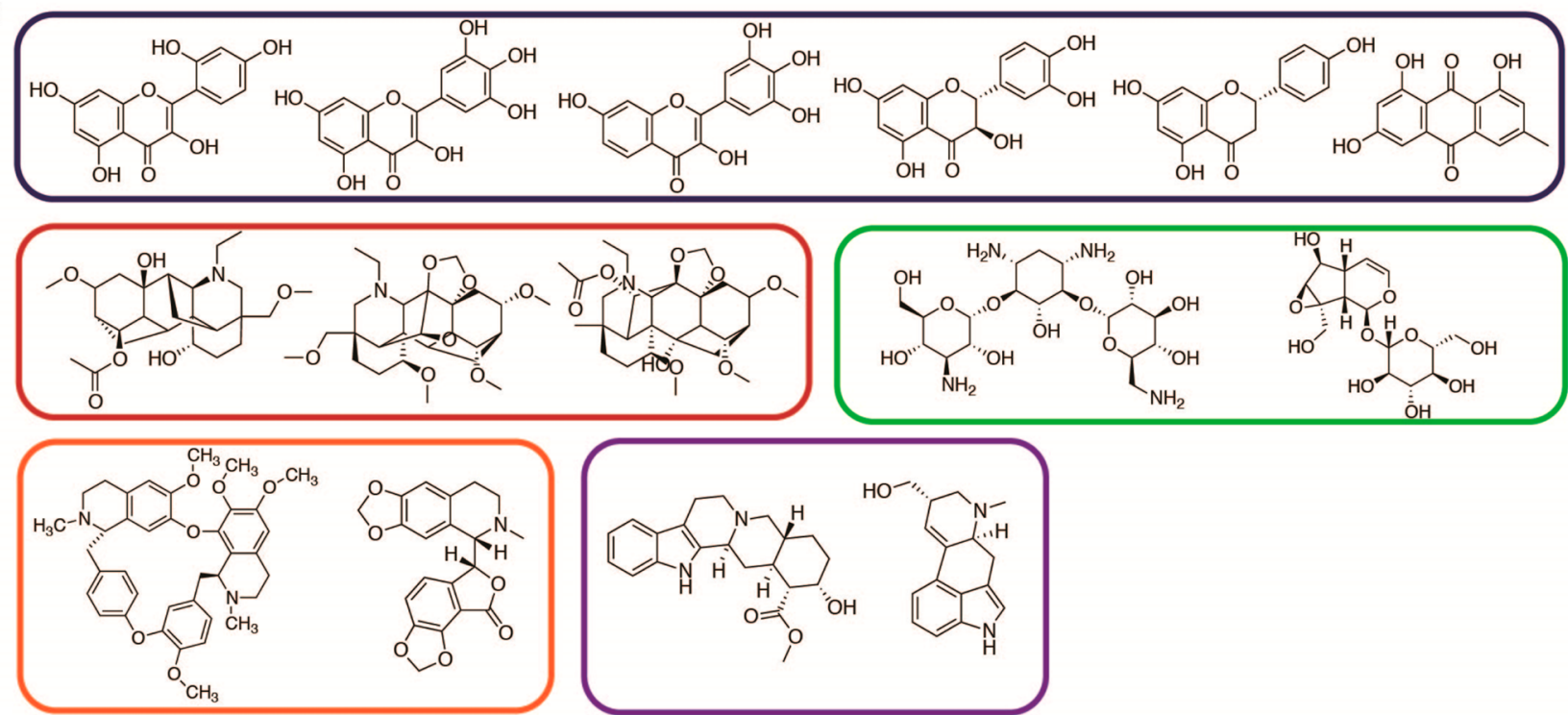

d

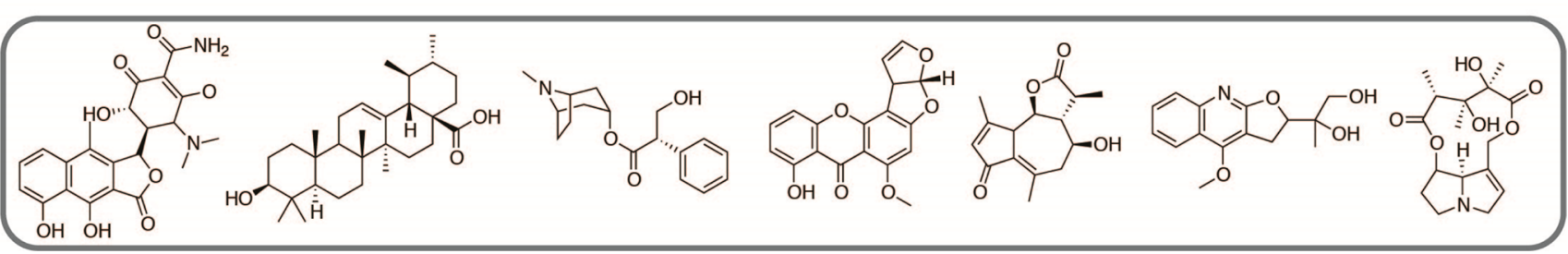

Figure 5. Structure similarity analysis of biocluster 1 with chemical similarity clusters highlighted. The similarity was assessed by Tanimoto similarity score using the extended connectivity fingerprint (ECFP) with bond diameter four (ECFP4) using Pipeline Pilot software (SciTegic/Accelrys). (a) Chemical similarity clustering at a Tc of 0.6. (b) Chemical similarity clustering at a Tc of 0.5 and 0.4 . (c) Chemical similarity clustering at a Tc of 0.3 . (d) Natural products remaining singletons at a Tc of 0.3 .

structures might act on different targets in a similar pathway or on different pathways leading to the same phenotype. Normal strategies employed in drug discovery, such as scaffold-hopping, would not identify these compounds. Valinomycin, in biocluster 1, is known to affect the lysosomal membrane pump. $^{34}$ Rapamycin (Rapamune), also in biocluster 1, targets mammalian target of rapamycin complex 1 (mTORC1) that is activated directly on the surface of the lysosomes. ${ }^{35}$ Therefore, perturbations in lysosomal function are consistent with compounds contained in this biocluster. The alteration of LysoTracker evident in multiple bioclusters may also reflect unique characteristics of the Parkinson's disease hONS cell line screened. We have shown previously that hONS cells derived form Parkinson's disease patients recapitulate some cellular and molecular aspects of the disease when compared to hONS cells from matched healthy controls. ${ }^{12,13}$ Interestingly, mutations in the vesicular trafficking protein VPS35 have been recently associated with late-onset Parkinson's disease. ${ }^{36,37}$ These mutations impair the lysosomal degradation of alpha-synuclein, which plays a central role in Parkinson's disease and neurotoxicity. ${ }^{38}$ The identification of compounds inducing similar or opposite phenotypes in Parkinson's disease patient- 
derived hONS cells might aid our understanding of vesicular trafficking pathways in Parkinson's disease.

All compounds profiled showed at least one phenotypic response that differs from DMSO-treated cells on the cell model. Phenotypic analysis showed that all the natural products in the data set have organelle effects that may arise from modulating human proteins or interactions with DNA or RNA. This study provides evidence that natural products occupy biologically relevant space, confirming that natural products are privileged structures with respect to recognition of biological space. The importance of profiling compounds in a doseresponse manner was found.

Primary cell models may present a more accurate picture of the in vivo state than immortalized or transformed cell models, as patient-derived cells preserve individual variability in RNA expression and cell function. ${ }^{12,13}$ Consequently, even though we observe overall similar biological profiles, we also expect to detect differing biological response to natural products when using cells from another patient/individual. We have shown here that cytological profiling is a sensitive but robust platform. As Parkinson's disease is a multifactorial disease, with a range of clinical presentations, this work ultimately aims to examine if there is a correlation between clinical records and cellular phenotypes in response to natural products. Comparing the phenotype with normal healthy individuals facilitates the possibility of a drug discovery approach using phenotypic reversal.

Further evidence that natural products occupy biologically relevant space has been provided by the observation of a phenotype for all studied natural products. The observation of a phenotype was improved by targeting multiple cellular compartments. However, chemical similarity did not drive the biological response. Unbiased multidimensional phenotypic screening may be useful as a starting point for drug discovery, by predicting the cellular function and subcellular site of activity of new compounds or by studying phenotype reversal.

\section{EXPERIMENTAL SECTION}

Compound Library. A total of 590 pure natural products derived from Nature Bank, a unique library established at the Eskitis Institute for Drug Discovery, were screened and profiled. Nature Bank contains a large diversity of known, new, and novel compounds isolated from plant and marine sources. In all cases, compounds were stored solubilized in DMSO at a stock concentration of $5 \mathrm{mM}$ and were determined by LC/MS and ${ }^{1} \mathrm{H}$ NMR to be a least $90 \%$ pure.

Compound Transfer. Compounds (10, 30, 100, and $300 \mathrm{~nL}$ ) were robotically transferred into two optically clear bottom CellCarrier 384-well plates (PerkinElmer). hONS cells from the Parkinson's disease cell line C1 200080013 were added to wells at a density of 1350 cells per well in $50 \mu \mathrm{L}$ of growth medium (DMEM/F12, 10\% FBS), leading to a final concentration of $1,3,10$, and $30 \mu \mathrm{M}(0.6 \%$ DMSO) for each compound. DMSO $(0.6 \%)$ was used as negative control. Rotenone (20 $\mu \mathrm{M}, 0.6 \% \mathrm{DMSO})$, chloroquine $(10 \mu \mathrm{M}, 0.6 \%$ DMSO), and nocodazole ( $10 \mu \mathrm{M}, 0.6 \%$ DMSO) were used in order to investigate the sensitivity and reproducibility of the method. The cells were incubated for $24 \mathrm{~h}$ at $37{ }^{\circ} \mathrm{C}$ under $5 \% \mathrm{CO}_{2}$.

Cell Staining. After $24 \mathrm{~h}$ of incubation with compounds, one 384well plate was treated with MitoTracker Orange CMTMRos (Invitrogen) $(400 \mathrm{nM})$ for $30 \mathrm{~min}$ at $37{ }^{\circ} \mathrm{C}, 5 \% \mathrm{CO}_{2}$. The second 384-well plate was treated with LysoTracker Red DND-99 (Invitrogen) $(100 \mathrm{nM})$ for $1 \mathrm{~h}$ at $37{ }^{\circ} \mathrm{C}, 5 \% \mathrm{CO}_{2}$. Cells were fixed in $4 \%$ paraformaldehyde for $5 \mathrm{~min}$ at room temperature (rt). Cells were washed twice with phosphate-buffered saline (PBS, SigmaAldrich) and treated with 3\% goat serum (Sigma-Aldrich) and $0.2 \%$ Triton X-100 (Sigma-Aldrich) in PBS for $45 \mathrm{~min}$ at rt. Plates were incubated with primary antibodies. Mouse anti- $\alpha$-tubulin (SigmaAldrich) and rabbit anti-LC3b (Sigma-Aldrich) were added to the plate already treated with MitoTracker, and mouse anti-EEA1 (SigmaAldrich) was added to the plate previously treated with LysoTracker. Plates were incubated at $\mathrm{rt}$ for $1 \mathrm{~h}$, then washed twice with PBS. Secondary antibodies goat anti-mouse Alexa-647 1/500 (Invitrogen) and goat anti-rabbit Alexa-488 1/500 (Invitrogen) were added to the first plate, and goat anti-mouse Alexa-488 1/500 (Invitrogen) was added to the second plate for $30 \mathrm{~min}$ at rt. Cells were washed twice with PBS and stained with $4^{\prime}, 6^{\prime}$-diamidino-2-phenylindole 1/5000 (Dapi, Invitrogen) and with CellMask Deep Red 1/5000 (Invitrogen) for the plate treated with LysoTracker and incubated for $10 \mathrm{~min}$ at rt. Cells were washed twice with PBS, and plates were stored in the dark at $4{ }^{\circ} \mathrm{C}$ with $25 \mu \mathrm{L}$ of $\mathrm{PBS} /$ well.

Imaging and Image Analysis. Plates were imaged automatically using Operetta (PerkinElmer), a high content imaging system using a $20 \times$ high numerical aperture objective lens. Six images per well for each wavelength were collected. Individual cell segmentation was done using the Harmony software, and measurements for each cell were performed generating 38 parameters from six dyes: Dapi, $\alpha$-tubulin staining, MitoTracker Orange CMTMRos, LC3b staining, LysoTracker Red DND-99, and EEA1 staining.

The normality of the data was checked for each parameter, and a $\log _{2}$ transform was made when required in order to perform a $t$ test. Then, the measurements were converted to $z$-scores, clustered using Cluster 3.0 software (uncentered correlation and centroid linkage), and analyzed using Java TreeView and Pipeline Pilot software.

Structure Similarity Search. The similarity of structures in each biocluster was assessed by Tanimoto similarity score using the extended connectivity fingerprint (ECFP) with bond diameter four (ECFP4) using Pipeline Pilot software (SciTegic/Accelrys). The structure similarity search was performed on the compounds that had all four concentrations in the same phenotypic cluster to avoid a bias due to dose-response activity.

\section{ASSOCIATED CONTENT}

\section{S Supporting Information}

The Supporting Information is available free of charge on the ACS Publications website at DOI: 10.1021/acs.jnatprod.6b00258.

List of the 38 biological parameters, trivial names of the compounds, Simplified Molecular Input Line Entry System (SMILES), and the biocluster concentrations/ structure similarity (PDF)

\section{AUTHOR INFORMATION}

\section{Corresponding Authors}

*E-mail: s.wood@griffith.edu.au.

*Tel: +61-7-3735-6000. Fax: +61-7-3735-6001. E-mail: r. quinn@griffith.edu.au.

\section{Notes}

The authors declare no competing financial interest.

\section{ACKNOWLEDGMENTS}

This research was supported under Australian Research Council's Discovery Projects funding scheme (project number DP130102400) and the Clem Jones Foundation. M.L.V. acknowledges Griffith University International Postgraduate Research Scholarships (GUIPRS).

\section{REFERENCES}

(1) Koch, M. A.; Waldmann, H. Drug Discovery Today 2005, 10, 471-483. 
(2) Koch, M. A.; Wittenberg, L. O.; Basu, S.; Jeyaraj, D. A.; Gourzoulidou, E.; Reinecke, K.; Odermatt, A.; Waldmann, H. Proc. Natl. Acad. Sci. U. S. A. 2004, 101, 16721-16726.

(3) Breinbauer, R.; Vetter, I. R.; Waldmann, H. Angew. Chem., Int. Ed. 2002, 41, 2878-2890.

(4) Dobson, C. M. Nature 2004, 432, 824-828.

(5) Harvey, A. L.; Edrada-Ebel, R.; Quinn, R. J. Nat. Rev. Drug Discovery 2015, 14, 111-129.

(6) Koehn, F. E.; Carter, G. T. Nat. Rev. Drug Discovery 2005, 4, 206-220.

(7) Kurita, K. L.; Linington, R. G. J. Nat. Prod. 2015, 78, 587-596.

(8) Schulze, C. J.; Bray, W. M.; Woerhmann, M. H.; Stuart, J.; Lokey, R. S.; Linington, R. G. Chem. Biol. 2013, 20, 285-295.

(9) Perlman, Z. E.; Slack, M. D.; Feng, Y.; Mitchison, T. J.; Wu, L. F.; Altschuler, S. J. Science 2004, 306, 1194-1198.

(10) Gustafsdottir, S. M.; Ljosa, V.; Sokolnicki, K. L.; Anthony Wilson, J.; Walpita, D.; Kemp, M. M.; Petri Seiler, K.; Carrel, H. A.; Golub, T. R.; Schreiber, S. L.; Clemons, P. A.; Carpenter, A. E.; Shamji, A. F. PLoS One 2013, 8, e80999.

(11) Tanaka, M.; Bateman, R.; Rauh, D.; Vaisberg, E.; Ramachandani, S.; Zhang, C.; Hansen, K. C.; Burlingame, A. L.; Trautman, J. K.; Shokat, K. M.; Adams, C. L. PLoS Biol. 2005, 3, e128.

(12) Matigian, N.; Abrahamsen, G.; Sutharsan, R.; Cook, A. L.; Vitale, A. M.; Nouwens, A.; Bellette, B.; An, J.; Anderson, M.; Beckhouse, A. G.; Bennebroek, M.; Cecil, R.; Chalk, A. M.; Cochrane, J.; Fan, Y.; Feron, F.; McCurdy, R.; McGrath, J. J.; Murrell, W.; Perry, C.; Raju, J.; Ravishankar, S.; Silburn, P. A.; Sutherland, G. T.; Mahler, S.; Mellick, G. D.; Wood, S. A.; Sue, C. M.; Wells, C. A.; Mackay-Sim, A. Dis. Models \&amp; Mech. 2010, 3, 785-798.

(13) Cook, A. L.; Vitale, A. M.; Ravishankar, S.; Matigian, N.; Sutherland, G. T.; Shan, J.; Sutharsan, R.; Perry, C.; Silburn, P. A.; Mellick, G. D.; Whitelaw, M. L.; Wells, C. A.; Mackay-Sim, A.; Wood, S. A. PLoS One 2011, 6, e21907.

(14) Grkovic, T.; Pouwer, R. H.; Vial, M. L.; Gambini, L.; Noél, A.; Hooper, J. N.; Wood, S. A.; Mellick, G. D.; Quinn, R. J. Angew. Chem., Int. Ed. 2014, 53, 6070-6074.

(15) Wang, D.; Feng, Y.; Murtaza, M.; Wood, S.; Mellick, G.; Hooper, J. N. A.; Quinn, R. J. J. Nat. Prod. 2016, 79, 353.

(16) Zhu, X.; Bidlingmaier, S.; Hashizume, R.; James, C. D.; Berger, M. S.; Liu, B. Mol. Cancer Ther. 2010, 9, 2131-2141.

(17) Stenmark, H.; Aasland, R.; Toh, B. H.; DArrigo, A. J. Biol. Chem.

1996, 271, 24048-24054.

(18) Lone, M.; Kungl, T.; Koper, A.; Bottenberg, W.; Kammerer, R.; Klein, M.; Sweeney, S. T.; Auburn, R. P.; O’Kane, C. J.; Prokop, A. J. Cell Sci. 2010, 123, 2369-2374.

(19) Peshenko, I. V.; Olshevskaya, E. V.; Dizhoor, A. M. J. Biol. Chem. 2008, 283, 21747-21757.

(20) Giuliano, K. A. J. Biomol. Screening 2003, 8, 125-135.

(21) Russell, J. W.; Golovoy, D.; Vincent, A. M.; Mahendru, P.; Olzmann, J. A.; Mentzer, A.; Feldman, E. L. FASEB J. 2002, 16, 17381748.

(22) Carpenter, J. E.; Jackson, W.; Benetti, L.; Grose, C. J. Virol. 2011, 85, 9414-9424.

(23) Farshori, P. Q.; Goode, D. J. Submicrosc. Cytol. Pathol. 1994, 26, 137-146.

(24) Michihara, A.; Toda, K.; Kubo, T.; Fujiwara, Y.; Akasaki, K.; Tsuji, H. Biol. Pharm. Bull. 2005, 28, 947-951.

(25) Li, N.; Ragheb, K.; Lawler, G.; Sturgis, J.; Rajwa, B.; Melendez, J. A.; Robinson, J. P. J. Biol. Chem. 2003, 278, 8516-8525.

(26) Camp, D.; Davis, R. A.; Campitelli, M.; Ebdon, J.; Quinn, R. J. J. Nat. Prod. 2012, 75, 72-81.

(27) Baldi, P.; Nasr, R. J. Chem. Inf. Model. 2010, 50, 1205-1222.

(28) Vogl, A. W.; Linck, R. W.; Dym, M. Am. J. Anat. 1983, 168, 99108.

(29) Schliwa, M. J. Cell Biol. 1982, 92, 79-91.

(30) Arnal, I.; Wade, R. H. Curr. Biol. 1995, 5, 900-908.

(31) Su, Y. F.; Li, S. K.; Li, N.; Chen, L. L.; Zhang, J. W.; Wang, J. R.

J. Med. Plants Res. 2011, 5, 5428-5432.
(32) Koppel, C.; Wagemann, A.; Martens, F. Eur. J. Drug Metab. Pharmacokinet. 1989, 14, 161-167.

(33) Lopez-Lazaro, M.; Palma; De La Pena, N.; Pastor, N.; MartinCordero, C.; Navarro, E.; Cortes, F.; Ayuso, M. J.; Toro, M. V. Planta Med. 2003, 69, 701-704.

(34) Harikumar, P.; Reeves, J. P. J. Biol. Chem. 1983, 258, 1040310410.

(35) Ohsaki, Y.; Suzuki, M.; Shinohara, Y.; Fujimoto, T. Histochem. Cell Biol. 2010, 134, 537-544.

(36) Vilarino-Guell, C.; Wider, C.; Ross, O. A.; Dachsel, J. C.; Kachergus, J. M.; Lincoln, S. J.; Soto-Ortolaza, A. I.; Cobb, S. A.; Wilhoite, G. J.; Bacon, J. A.; Behrouz, B.; Melrose, H. L.; Hentati, E.; Puschmann, A.; Evans, D. M.; Conibear, E.; Wasserman, W. W.; Aasly, J. O.; Burkhard, P. R.; Djaldetti, R.; Ghika, J.; Hentati, F.; KrygowskaWajs, A.; Lynch, T.; Melamed, E.; Rajput, A.; Rajput, A. H.; Solida, A.; Wu, R. M.; Uitti, R. J.; Wszolek, Z. K.; Vingerhoets, F.; Farrer, M. J. Am. J. Hum. Genet. 2011, 89, 162-167.

(37) Follett, J.; Norwood, S. J.; Hamilton, N. A.; Mohan, M.; Kovtun, O.; Tay, S.; Zhe, Y.; Wood, S. A.; Mellick, G. D.; Silburn, P. A.; Collins, B. M.; Bugarcic, A.; Teasdale, R. D. Traffic 2014, 15, 230-244.

(38) Stefanis, L. Cold Spring Harbor Perspect. Med. 2012, 2, a009399. 